\title{
Studies of Cosmic-Ray Proton Flux with the DAMPE Experiment
}

\author{
Chuan Yue ${ }^{* 1,2}$, Jing-Jing Zang ${ }^{1}$,Tie-Kuang Dong ${ }^{1}$, Antonio Surdo ${ }^{3,4}$, Stefania \\ Vitillo $^{5}$, on behalf of the DAMPE collaboration ${ }^{\dagger}$ \\ ${ }^{1}$ Key Laboratory of Dark Matter and Space Astronomy, Purple Mountain Observatory, Chinese \\ Academy of Sciences, Nanjing 210008, China \\ ${ }^{2}$ University of Chinese Academy of Sciences, Beijing 100049, China \\ ${ }^{3}$ Università del Salento - Dipartimento di Matematica e Fisica "E. De Giorgi”, I-73100, Lecce, \\ Italy \\ ${ }^{4}$ Istituto Nazionale di Fisica Nucleare (INFN) - Sezione di Lecce, I-73100, Lecce, Italy \\ ${ }^{5}$ Department of Nuclear and Particle Physics, University of Geneva, CH-1211, Switzerland \\ E-mail: yuechuanepmo.ac.cn
}

\begin{abstract}
The DArk Matter Particle Explorer (DAMPE) is a space-based mission designed as a high energy particle detector for measuring cosmic-rays and gamma-rays in space. It was successfully launched on December 17, 2015, and since then it has been in stable data taking for more than one year. The large geometric factor and thick calorimeter enables DAMPE to have very good potential to measure cosmic-rays from $20 \mathrm{GeV}$ up to $100 \mathrm{TeV}$. Here we analyze the proton component using the DAMPE flight data. Comprehensive Monte-Carlo simulations have been employed to validate the event selections and estimate the efficiency as well as the geometry factor. The analysis progress is reported and discussed.
\end{abstract}

35th International Cosmic Ray Conference - ICRC2017

12-20 July, 2017

Bexco, Busan, Korea

\footnotetext{
*Speaker.

$\dagger$ The DAMPE mission is funded by the strategic priority science and technology projects in space science of Chinese Academy of Sciences. In China the data analysis is supported in part by the National Key Research and Development Program of China (No. 2016YFA0400200), National Basic Research Program of China (No. 2013CB837000), National Natural Science Foundation of China (Nos. 11525313, 11622327, 11673075, 11303107 and 11673047), and the 100 Talents Program of Chinese Academy of Sciences. In Europe the activities and the data analysis are supported by the Swiss National Science Foundation (SNSF), Switzerland; the National Institute for Nuclear Physics (INFN), Italy.
} 


\section{Introduction}

Cosmic rays (CRs) with energies up to the so-called "knee" (at $E \sim 4 \mathrm{PeV}$ [1] where the all-particle spectrum exhibits a spectral softening) are widely believed to be accelerated by strong shocks due to e.g., the supernova explosions. Protons are the most abundant particles in the primary CRs. Precise measurements of the energy spectrum of protons up to PeV energies are essential in understanding the origin and acceleration of them, as well as the physics of the "knee" [2].

While at low energy $(E \lesssim 30 \mathrm{GeV})$ CRs are significantly affected by the solar modulation, the energy spectrum of CRs above a few tens of $\mathrm{GeV}$ according to traditional acceleration and propagation models is expected to follow a featureless power-law. Several recent measurements of the proton flux by balloon-borne experiment ATIC-2 [3] and space-borne experiments PAMELA [4] and AMS-02 [5] observed a deviation (hardening) from single power-law distribution at rigidities of $\sim 300 \mathrm{GV}$. Similar behaviors are also shown in the spectra of heavier nuclei [6]. These results challenge the conventional picture of the CR production and propagation. Various types of modification of the conventional model have been proposed (e.g., [7, 8, 9, 10]).

The precise measurement of $\mathrm{CR}$ spectrum between $\mathrm{TeV}$ and $\mathrm{PeV}$ energies is crucial for discriminating different models to explain the spectral hardening. Current direct measurements have relatively large uncertainties for $E>\mathrm{TeV}$, due to limited counting statistics from balloon- or spaceborne detectors. The statistics is no longer an issue for ground-based air shower experiments. However, the high energy threshold and poor composition resolution of air shower experiments make the measurements difficult and largely dominated by systematics. The DArk Matter Particle Explorer (DAMPE) is a calorimetric-type, satellite-borne detector for high energy electron, $\gamma$-ray, and CR observations. The DAMPE detector has a large geometric factor, and is expected to improve significantly the direct measurement of the proton spectrum above $\mathrm{TeV}$ [11].

\section{DAMPE instrument}

The DAMPE detector consists of 4 sub-detectors, which are a Plastic Scintillator strip Detector (PSD), a Silicon-Tungsten tracKer-converter (STK), a BGO imaging calorimeter, and a NeUtron Detector (NUD) from top to bottom [11]. The PSD measures the charge of an incident particle. It can also be used as an anti-coincidence detector for $\gamma$-rays. The STK reconstructs the trajectory and measures the charge. The BGO calorimeter measures the energy, and provides electron-hadron identification. The track information can also be obtained via the BGO image. The NUD provides additional electron-hadron discrimination, which is important for energies above TeV. These 4 sub-detectors enable good measurements of the charge $(|Z|)$, arrival direction, energy, and particle identity of each event. The major scientific objectives of DAMPE consist of indirect search for particle dark matter, $\gamma$-ray astronomy, and studies on origin and propagation of Galactic CRs. For more details about the instrument and its performance please refer to Refs. [11, 12, 13, 14].

\section{Data analysis}

Seventeen months of DAMPE on-orbit data from January $1^{s t}, 2016$ to May 31 ${ }^{s t}, 2017$ are analyzed in this work. The live time is estimated precisely by accumulating the lost time with 
event-by-event basis, which consists of three parts. The first part refers to the time in South Atlantic Anormaly (SAA) region, which counts on average $4.5 \%$ of the operation time; The second part comes from the instrumental dead time, which imposes $3.0725 \mathrm{~ms}$ per event; And the third one is due to the on-orbit calibration data-taking which takes about $1.5 \%$ of time. The overall exposure time during the 17 months period is $3.414 \times 10^{7}$ seconds, corresponding to a fraction of $76.44 \%$.

\subsection{Event Selection}

To ensure that all detectors work in good condition, data recorded when the satellite was in the SAA region are excluded. Furthermore, we select events with deposited energy in the calorimeter larger than $20 \mathrm{GeV}$, to effectively avoid the effect due to the geomagnetic rigidity cutoff [15].
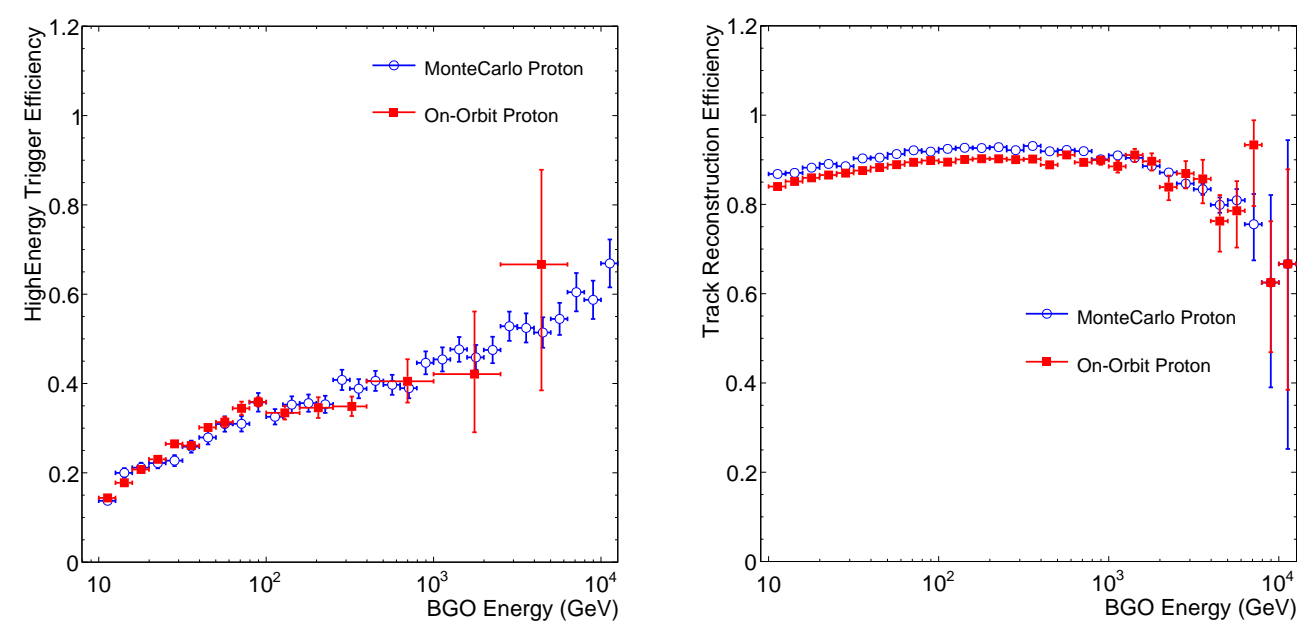

Figure 1: Left - The HE trigger efficiency of protons as a function of BGO energy for flight data (red) and MC data (blue). Right — The track reconstruction efficiency for flight data (red) and MC data (blue).

- Pre-selection

The events are required to pass the High-Energy (HE) trigger in order to reject the minimum ionizing particles (MIPs). The HE trigger efficiency is estimated by means of unbias trigger sample, which is pre-scaled by $1 / 512$ at low latitudes $\left(\leqslant \pm 20^{\circ}\right)$ and $1 / 2048$ at high latitudes:

$$
\varepsilon_{\text {trigger }}=\frac{N_{\text {he|unb }}}{N_{\text {unb }}}
$$

where $N_{\text {unb }}$ is the number of events which pass the proton selection and are triggered as unbiased sample, and $N_{\text {he unb }}$ is the number of events which pass the HE trigger in the selected unbiased sample $N_{\text {unb }}$. The left panel of Figure 1 shows the HE trigger efficiency as a function of deposit energy in the BGO calorimeter (BGO energy). For energies higher than hundreds of $\mathrm{GeV}$, the uncertainty of flight data increases due to the limited statistics of the unbiased sample. The difference between the flight data and the Monte-Carlo (MC) simulation is found to be within $\sim 8.0 \%$ below $200 \mathrm{GeV}$, which is taken as one kind of systematic errors. Apart from the HE trigger, the pre-selection requires at least one hit in each sub-layer of the PSD and one good track in the STK with more than four hits in both $X$ and $Y$ views. 
- Track selection

In case that there are more than one good tracks in the STK, the most reliable one is selected by jointly considering the following factors: the length of the track, the reduced $\chi^{2}$ value of the fit, and the match between the candidate track and the shower axis in the calorimeter. The selected track is then required to cross all sub-layers of the PSD and pass through the calorimeter from top to bottom. To estimate the STK track efficiency for proton, a pure proton sample is obtained by applying an independent charge selection based on the reconstructed shower axis in BGO. In this case, the track efficiency is estimated as the ratio of the number of events after the proton track selection over the number of events. The right panel of Figure 1 shows the track reconstruction efficiency as a function of BGO energy. The results of MC data and flight data are consistent with each other within $2.5 \%$ up to $10 \mathrm{TeV}$.

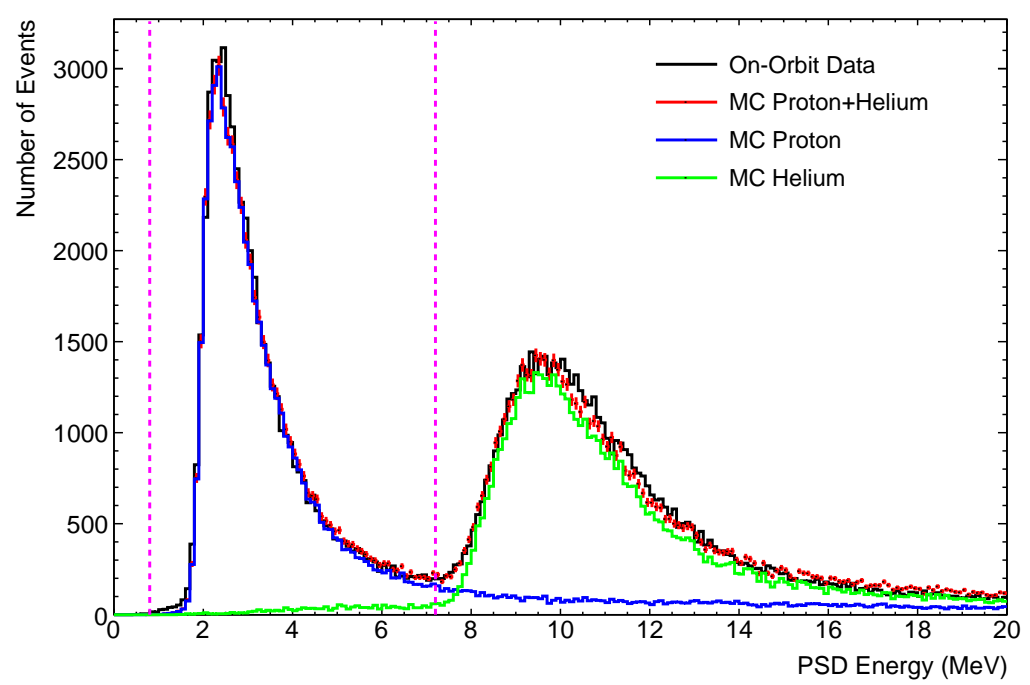

Figure 2: The combined energy spectrum of PSD for protons and helium nuclei, for BGO energies between 445 and $560 \mathrm{GeV}$. The on-orbit data (black) are compared with the best-fit templates of simulations for protons (blue), helium nuclei (green), and their sum (red). Vertical dashed lines show the cuts to select proton candidates.

- Charge selection

The PSD of DAMPE is responsible for the charge measurement of CR nuclei up to $Z=26$. The PSD is composed of two layers placed in a hodoscopic configuration ( $Y$-view for layer1 and $X$-view for layer-2), with 41 plastic scintillator strips in each layer [12]. The deposit energy in a hit strip due to ionization is proportional to $Z^{2}$, which is about $2 \mathrm{MeV}$ for protons. The charge is measured independently by the two PSD layers. The combined energy (and hence charge) spectrum of PSD is plotted in Figure 2, where the proton and helium peaks can be clearly seen. Protons are selected through BGO energy-dependent cuts of the PSD energy (e.g., PSD energy in [0.6,7.2] MeV for $445-560 \mathrm{GeV}$ of BGO energies as shown in Figure 2) to enable a relatively high proton efficiency and a low helium background in the whole energy range. The charge reconstruction efficiency is estimated independently 
for each PSD layer, as shown in Figure 3. The deviation of the flight data from the MC simulations is within $2 \%$ for layer- 1 and $4 \%$ for layer- 2 .
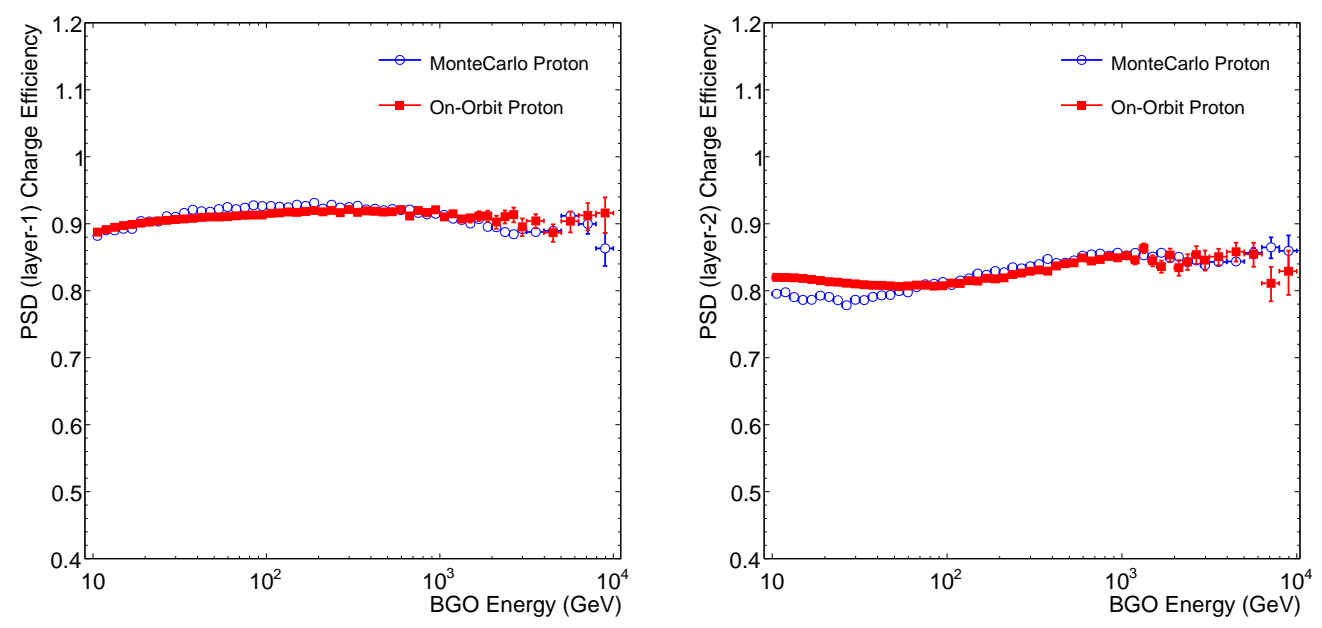

Figure 3: The charge reconstruction efficiency of protons as a function of BGO energy for PSD layer 1 (left) and layer 2 (right). The flight data (red) are compared with the MC data (blue).

\subsection{Background}

The background for protons includes mis-identified helium nuclei and a tiny fraction of electrons. Given the very good e/p separation power of DAMPE, electrons can be largely rejected [11]. The fraction of residual electrons in the proton sample is estimated to be $\lesssim 0.1 \%$ for deposit energies of decades of $\mathrm{GeV}$. Helium nuclei represent the second most abundant CR component and serve as the main source of background in proton sample. To estimate the helium contamination, a template-fit method is applied to the charge distributions. The templates are obtained through MC simulations (see Figure 2). The helium contamination as a function of deposit energy in the BGO calorimeter is shown in Figure 4. We can see that the helium background is within 2.0\% up to 10 $\mathrm{TeV}$.

\subsection{Effective Acceptance}

The effective acceptance is defined as the product of the geometric factor and selection efficiencies (including trigger, track, and charge selections). To estimate the effective acceptance, a detailed MC simulation using the GEANT4 toolkit [16] is performed. A digitization algorithm including the DAMPE detector response is developed for converting the raw energy hits by GEANT4 simulations into Analog-to-Digital Converter (ADC) counts. Then we apply the reconstruction algorithm to the simulation data to obtain the reconstructed events [17]. An isotropic, single powerlaw proton spectrum is simulated and then the effective acceptance of the $i-$ th primary energy bin is obtained by

$$
A_{\text {eff }, i}=A_{\text {gen }} \times \frac{N_{\text {pass }, i}}{N_{\text {gen }, i}}
$$

where $A_{\text {gen }}$ is the geometrical factor of the MC generation sphere, $N_{\text {gen }, i}$ and $N_{\text {pass }, i}$ are the numbers of generated events and those passing the selections. The effective acceptance as a function of 


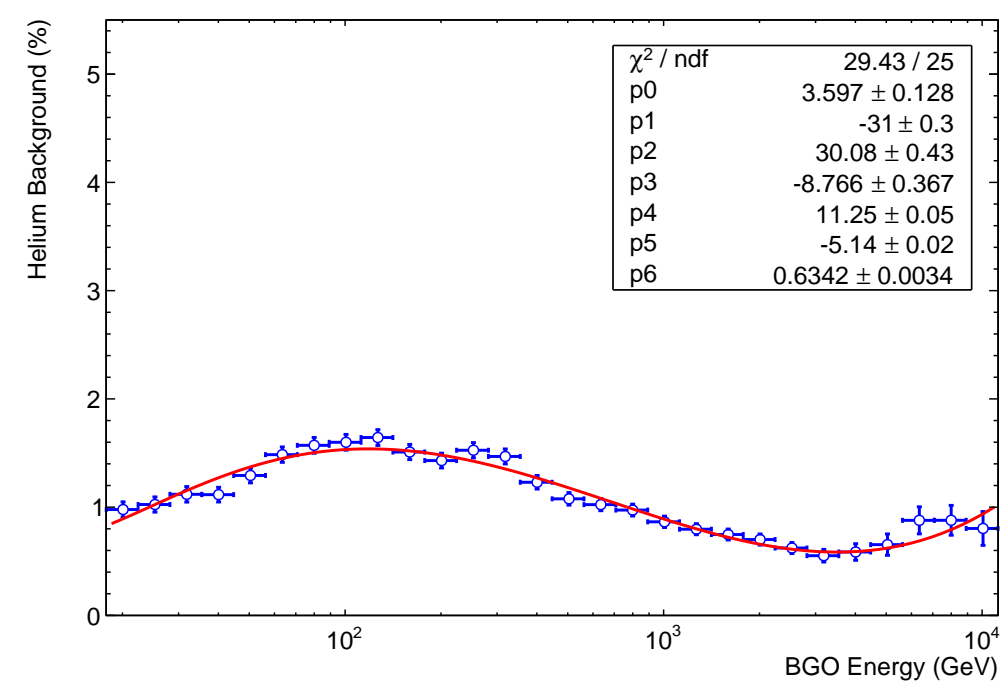

Figure 4: The fraction of helium background in proton candidate events as a function of BGO energy.

primary energy is shown in Figure 5. The systematic error on the effective acceptance, $\sigma_{\text {effacc }}$, is estimated as

$$
\sigma_{\text {effacc }}=\sqrt{\sigma_{\text {trigger }}^{2}+\sigma_{\text {track }}^{2}+\sigma_{\text {charge }}^{2}} \approx 9.5 \%,
$$

where $\sigma_{\text {trigger }} \approx 8.0 \%$ is the systematic error on the trigger efficiency, $\sigma_{\text {track }} \approx 2.5 \%$ is the systematic error on the track reconstruction efficiency, and $\sigma_{\text {charge }} \approx 4.5 \%$ is the systematic error on the charge reconstruction efficiency.

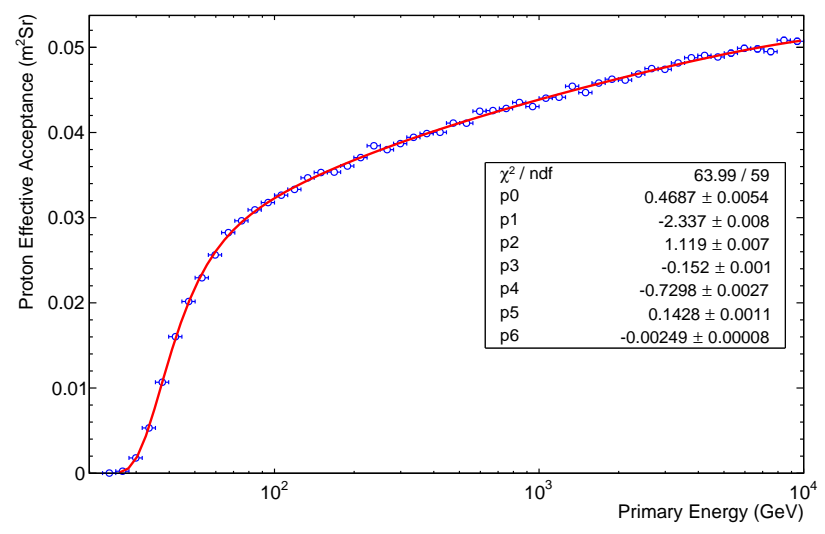

Figure 5: The effective acceptance of proton as a function of primary energy. The red line represents the fit result with an experimental function.

\subsection{Energy Unfolding}

Due to the limited thickness of the BGO calorimeter ( $~ 1.62$ nuclear length) and the nondetection of muon and neutrino components in a hadronic showers, which brings large uncertainty 
of the energy measurement, a CR nucleus does not deposit its energy fully in the calorimeter. As a consequence, the energy resolution for protons in DAMPE is $\sim 15 \%$ at $100 \mathrm{GeV}$ and $\sim 25 \%$ at $10 \mathrm{TeV}$ [11]. To convert the measured energy spectrum to the primary energy spectrum, it is necessary to unfold the instrument response. Instead of correcting the particle energy event-byevent, the unfolding procedure enables an estimate of the energy distribution of incident particles from the deposit energy distribution. The response matrix was obtained with MC simulations and parametrized with emprical functions. The systematic error due to the unfolding procedure is estimated by varying the parametrization of the response matrix, which is found to be less than $1.5 \%$ below $100 \mathrm{GeV}$ and $\sim 4.2 \%$ at $10 \mathrm{TeV}$.

\section{Results and discussion}

Figure 6 shows the preliminary proton flux as a function of kinetic energy measured with DAMPE, compared with previous measurements by PAMELA [4], ATIC-2 [3], AMS-02 [5] and CREAM-III [18]. The statistical errors are ploted as error bars while the systematic errors are showed with a band. Note that for the CREAM-III data we also add $\sim 10 \%$ uncertainties, primarily due to the uncertainty from the energy scale [18]. Our results show good agreement with the AMS02 and PAMELA data for $E_{k} \lesssim 200 \mathrm{GeV}$. The spectral hardening at $E_{k} \gtrsim 200 \mathrm{GeV}$ can be observed from our measurements, confirming the previous results.

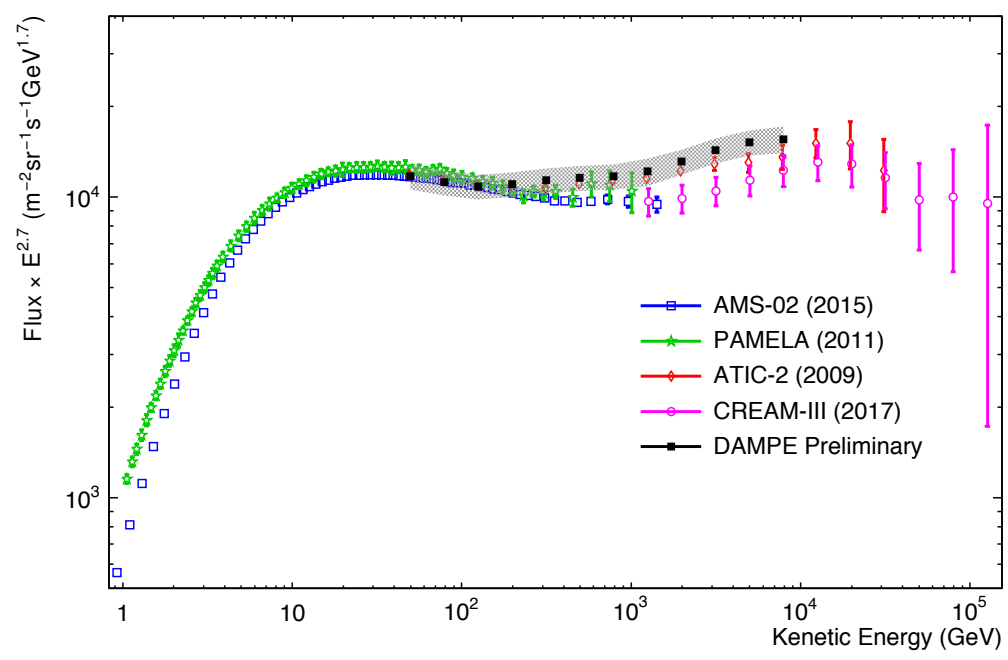

Figure 6: The preliminary $E_{k}^{2.7}$ weighted flux of CR protons measured by DAMPE, compared with previous results by PAMELA [4], AMS-02 [5], ATIC-2 [3], and CREAM-III [18]. The error bars represent statistical errors and the grey band shows the systematic errors.

More detailed analysis of the DAMPE data and more precise estimation of systematic uncertainties are on-going. With the accumulation of data, DAMPE is expected to measure the proton spectrum up to $100 \mathrm{TeV}$ with high precision, which will represent a significant step forward in understanding the origin and acceleration of CRs. 


\section{References}

[1] M. Amenomori et all, [The Tibet AS $\gamma$ Collaboration] The All-Particle Spectrum of Primary Cosmic Rays in the Wide Energy Range from $10^{14}$ to $10^{17} \mathrm{eV}$ Observed with the Tibet-III Air-Shower Array, Astrophys. J. 678 (2008) 1165

[2] T. K. Gaisser, T. Stanev and S. Tilav, Cosmic Ray Energy Spectrum from Measurements of Air Showers, Front. Phys. 8 (2013) 748 [arXiv:1303.3565].

[3] A. D. Panov et al., Energy Spectra of Abundant Nuclei of Primary Cosmic Rays from the Data of ATIC-2 Experiment: Final Results, Bull. Russ. Acad. Sci. Phys. 73 (2009) 564 [arXiv:1101.3246].

[4] O. Adriani et al. [PAMELA Collaboration], PAMELA Measurements of Cosmic-ray Proton and Helium Spectra, Science 332 (2011) 69 [arXiv:1103.4055].

[5] M. Aguilar et al. [AMS Collaboration], Precision Measurement of the Proton Flux in Primary Cosmic Rays from Rigidity $1 \mathrm{GV}$ to $1.8 \mathrm{TV}$ with the Alpha Magnetic Spectrometer on the International Space Station, Phys. Rev. Lett. 114 (2015) 171103.

[6] H. S. Ahn et al., Discrepant hardening observed in cosmic-ray elemental spectra, Astrophys. J. Lett. 714 (2010) L89 [arXiv:1004.1123].

[7] Y. Ohira and K. Ioka, Cosmic Ray Helium Hardening, Astrophys. J. Lett. 729 (2011) L13 [arXiv:1011.4405].

[8] Q. Yuan, B. Zhang and X. J. Bi, Cosmic ray spectral hardening due to dispersion in the source injection spectra, Phys. Rev. D 84 (2011) 043002 [arXiv:1104.3357].

[9] A. E. Vladimirov, G. e. Johannesson, I. V. Moskalenko and T. A. Porter, Testing the Origin of High-Energy Cosmic Rays, Astrophys. J. 752 (2012) 68 [arXiv:1108.1023].

[10] N. Tomassetti, Origin of the Cosmic-Ray Spectral Hardening, Astrophys. J. Lett. 752 (2012) L13 [arXiv:1204.4492].

[11] J. Chang et al. [DAMPE Collaboration], The Dark Matter Particle Explorer mission, Astropart. Phys. submitted (2017). [arXiv:1706.08453].

[12] Y. Yu et al., The Plastic Scintillator Detector at DAMPE, arXiv:1703.00098

[13] P. Azzarello et al., The DAMPE silicon-tungsten tracker, Nucl. Instrum. Meth. A 831 (2017) 379

[14] Z. Zhang et al., The calibration and electron energy reconstruction of the BGO ECAL of the DAMPE detector, Nucl. Instrum. Meth. A 836 (2016) 98 [arXiv:1602.07015].

[15] E. Thébault et al., International Geomagnetic Reference Field: the 12th generation, Earth, Planets and Space 67 (2017) 79

[16] S. Agostinelli, et al., Geant4 - a simulation toolkit Nucl. Instrum. Meth. A 506 (2017) 3

[17] C. Yue et al., A Parameterized Energy Correction Method for Electromagnetic Showers in BGO-ECAL of DAMPE, Nucl. Instrum. Meth. A 856 (2017) 11 [arXiv:1703.02821].

[18] Y. S. Yoon et al., Proton and Helium Spectra from the CREAM-III Flight, Astrophys. J. 839 (2017) no.1, 5 [arXiv:1704.02512]. 